\title{
Sucrose in the Concentrated Solution or the Supercooled "State": A Review of Caramelisation Reactions and Physical Behaviour
}

\author{
M. A. C. Quintas · J. F. Fundo • C. L. M. Silva
}

Received: 7 January 2010/Accepted: 31 March 2010/Published online: 22 April 2010

(C) Springer Science+Business Media, LLC 2010

\begin{abstract}
Sucrose is probably one of the most studied molecules by food scientists, since it plays an important role as an ingredient or preserving agent in many formulations and technological processes. When sucrose is present in a product with a concentration near or greater than the saturation point-i.e. in the supercooled state-it possesses high potentialities for the food industry in areas as different as pastry industry, dairy and frozen desserts or films and coatings production. This paper presents a review on critical issues and research on highly concentrated sucrose solutions - mainly, on sucrose thermal degradation and relaxation behaviour in such solutions. The reviewed works allow identifying several issues with great potential for contributing to significant advances in Food Science and Technology.
\end{abstract}

Keywords Supercooled sucrose - Relaxation behaviour . Caramelisation reaction

\section{Introduction}

Sucrose and its supercooled solutions are of utmost importance in areas as different as food and pharmaceutical

\footnotetext{
M. A. C. Quintas $(\bowtie)$. J. F. Fundo · C. L. M. Silva Laboratório de Optimização de Processos Alimentares, CBQF/ Escola Superior de Biotecnologia, Universidade Católica Poruguesa, Rua Dr Antonio Bernardino de Almeida, 4200-072 Porto, Portugal

e-mail: maquintas@mail.esb.ucp.pt

M. A. C. Quintas

Institute for Biotechnology and Bioengineering (IBB), Centre of Biological Engineering, Universidade do Minho, Campus de Gualtar, 4710-057 Braga, Portugal
}

industries or cryopreservation of biological systems. It is probably one of the most studied molecules by food scientists, since it plays an important role as an ingredient or preserving agent in many formulations and technological processes.

When sucrose is present in a product with a concentration near or greater than the saturation point-i.e. in the supercooled state-it possess high potentialities for the food industry in areas as different as pastry industry, dairy and frozen desserts or films and coatings production.

The use of sucrose concentrated solutions on confectionery is considered by many as an art only mastered by great chefs in trendy restaurants, or older women who devoted their lives to cooking in small pastry shops located in small villages. However, this could not be farther from the truth: there is a lot of science behind the production of sugar syrups. Such production involves the use of high temperatures with subsequent water evaporation. The extent of sugar degradation and the final water content in sugar syrups are critical parameters that influence aroma, sweet taste, mouthfeel and texture of the final pastry products [92]. Sweet creams, produced essentially with egg yolk and sugar are an example of the importance of concentrated sucrose solutions in pastry [49]. This kind of product can be used in pastry and toppings, or consumed as a sweet dessert, and also a basis for production of several different desserts, like fruit and nut custards. In order to provide the typical structure, colour, aroma, smoothness and consistency of this kind of product, sugar syrups have a crucial importance since it viscosity influences all these characteristics.

The use of amorphous sucrose in coating or infusion of nut products is also a traditional application of supercooled sucrose. Furthermore, amorphous sucrose is important in frozen food products, especially in ice creams [55, 57]. In 
these products, control of ice crystal growth is important for stability during storage and consumer acceptance. In most cases, increased sugars concentration is able to control or even reduce ice crystallisation [57, 120].

Sucrose has also been studied for its plasticising effect in films, including plasticising fish myofibrillar proteins to produce packaging biomaterials by thermal compressionmoulding [35] or, more recently in edible coatings applied to fresh cut carrots slices [133], and in white asparagus during cold storage [130]. Also, sucrose was used as coating ingredient for a method of encapsulation purified egg yolk immunoglobulins from immunised hens using a fluidised bed granulator [10].

This paper aims at reviewing the main aspects related to physical-chemical properties of supercooled sucrose. More specifically, of the relevant research that contributed to the development of a deeper understanding of chemical and physical properties of such supersaturated solutions. Namely:

1. The effect of heat treatment of sucrose in high concentrations and the kinetics of caramelisation reaction;

2. The physical behaviour of supersaturated sucrose solutions;

3. The systematisation and understanding of the underlying phenomena in these systems, by means of different mathematical modelling approaches.

\section{The Effect of Heat Treatment on Supercooled Sucrose}

\section{The Caramelisation Reaction}

\section{Reaction Mechanism}

Sucrose thermal degradation is an important reaction in the food industry, since it is responsible for either important characteristics of the final food products or can influence the yield of obtainable white sugar in sugar manufacture. This degradation may occur by two different major reaction pathways: the Maillard reaction, which takes place in the presence of amino acids, and caramelisation, that occurs when sucrose is heated at high temperatures [16].

Caramelisation, which is the common name for a group of complex reactions, has been studied by several authors. In the past decades, great insight was gained on the reactions mechanism $[32,84,106]$.

The caramelisation reaction occurs in the presence or absence of catalysts (acids, basis or salts and impurities). It can be observed in diluted or concentrated solutions (amorphous state), and in the crystal form, leading to many different pathways. Each possible pathway may be predominant under given environmental conditions. In this review, we focus on the case where sucrose in very concentrated solutions (above saturation at room temperature) is heated at high temperatures in the absence of impurities and at neutral $\mathrm{pH}$. Under these conditions, the effect of heat is similar to the effect of using lower temperatures in acidic conditions and the reaction mechanisms can be considered to be identical [84]. This is due to the formation of weak acids during sucrose hydrolysis.

The first step in the caramelisation reaction is sucrose hydrolysis (please see Fig. 1a). This takes place by protonation of the glycosidic linkage $[32,42,84,106]$. The $\mathrm{H}^{+}$ used in this reaction step can be derived from water (or even sucrose) dissociation at high temperatures and also from acidic reaction products $[42,76,106]$.

Under neutral conditions, sucrose hydrolysis yields D-glucose and a fructose oxocarbonium ion [32, 42, 76, 106]. This fructose carbocation can react with: (1) a water molecule to form fructose and regenerate the catalyst $\mathrm{H}^{+}$, or (2) another carbohydrate molecule present in solution (sucrose, glucose, fructose or even another fructose carbocation) to form oligosaccharides. Moreover, this fructose carbocation may undergo non-specific reactions to form a wide range of products, including: anhydrosaccharides, 5-hydroxymethyl-furfural (HMF) and organic acids [32, 42, 106].

The oligosaccharide and anhydrosaccharide components of caramel have been extensively studied $[38,39,80-82$, 102]. However, the extent of production of these substances is dependent on reaction conditions and is typically favoured by extreme acidic/high temperatures reaction conditions $[5,81,82]$.

Further degradation of fructose and glucose is responsible for the formation of other compounds, especially to HMF [5, 32, 42, 70, 76, 84, 126]. The mechanism of such reaction is generally accepted to be an open chain $\beta$-elimination mechanism, which proceeds via an enediol intermediate to 3-deoxyhexulose $[32,70]$. With the further development of the caramelisation reaction, HMF can be degraded to other products, including: (1) furfural, via fragmentation reaction (with indirect subsequent $\mathrm{H}^{+}$ release) and (2) 5-methylfurfural, via reduction reaction $[5,32,70,84]$.

The simplified mechanism of sucrose caramelisation reaction at high temperatures and neutral $\mathrm{pH}$ can then be generally described by the scheme in Fig. 1a).

\section{Colour Changes During Sucrose Thermal Degradation}

Non-enzymatic browning is the general cause of a food product darkening due to any reaction not owed to enzymatic activity. Although this includes several reaction types, such as lipid oxidation or ascorbic acid degradation, 
Fig. 1 Schematic representation of sucrose caramelisation mechanism: a based on literature search and b proposed reaction pathways based on experimental data and multiresponse modelling procedures [96]
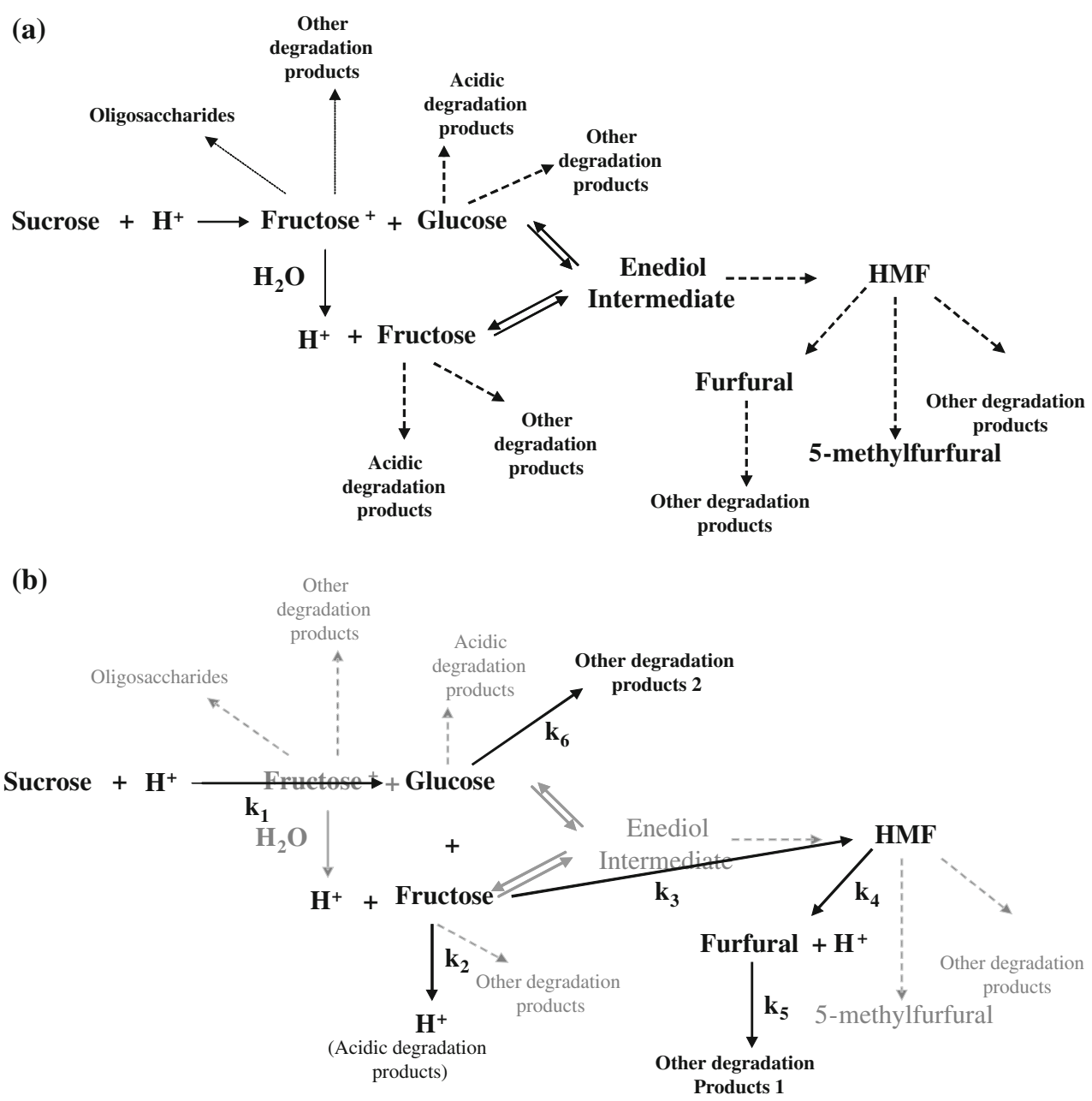

non-enzymatic browning is mainly associated with carbohydrate degradation reactions, such as the Maillard and caramelisation reactions [16].

Colour changes due to the Maillard reaction are extensively described in literature $[11,14,24,37,64,75,105$, 113]. On the other hand, caramelisation-related colour development has been less studied [23, 25].

The typical brown colour developed during caramelisation is attributed to the production of polymeric products during the reaction course. HMF and furfural are pointed out as precursors of such polymers [70].

Colour changes together with HMF content have been studied for numerous food systems, such as fruit juices and purees $[19,30,50,61,66,103,129]$, dairy products $[27$, $71,86]$, baked goods [98, 99], infant foods [46, 53, 101], honey [127] and model Maillard systems [28, 29, 118].

However, few studies have tried to correlate HMF content with colour development. Most of such studies only report the observation or not of a linear correlation between such factors [26, 45]. In even fewer cases, the possibility of an exponential correlation is addressed [100], and one study reported the use of a power law to describe such correlation [74]. Recently, it was shown that colour changes during caramelisation reaction presented and autocatalytic behaviour similar to sucrose degradation kinetics under the same conditions. Moreover, the contribution of HMF content to colour changes can be described by a fractional conversion model (for $\mathrm{L} / \mathrm{L}_{0}$ ) or a power law model (for total colour difference) [97].

\section{Caramelisation Reaction Kinetics}

\section{Sucrose Degradation Behaviour}

The Maillard reaction kinetics has been widely studied in the food science field [11, 25, 64, 132]. Caramelisation, on the other hand, is considered to follow a more simple reaction pathway and, consequently, fewer studies have been dedicated to its kinetics.

Caramelisation reaction is influenced by $\mathrm{pH}$, impurities (e.g. salts or other sugars like glucose or fructose) and solution's sucrose concentration. Several authors approached these aspects [32, 43, 76, 84, 106, 135]. Most of those works are in a range of low sucrose concentration $(<70 \%$ 
$(w / w))$, relatively low processing temperatures $\left(<85^{\circ} \mathrm{C}\right)$, presence of impurities and/or acidic or alkaline media [84]. A first-order model is often used to describe the heat degradation of sucrose under such conditions [25, 135].

There is little information on the kinetics of sucrose thermal degradation at high concentrations. A study was carried under these conditions, the research was related to acidic saturated solutions and a first-order reaction was observed [114].

However, in some cases, like specific confectionery products [36] or sugar boiling during white sugar production [106], high concentrated sucrose solutions are heated at high temperatures and neutral $\mathrm{pH}$. In such cases, a lag phase on sucrose degradation occurs [32, 42, 76]. This behaviour has been modelled assuming a pseudo-first-order reaction for concentrated sucrose thermal degradation in the presence of different salts [42]. A lag phase was also observed in a study of cane juice stored at factory room temperature, where the sucrose degradation was due to the combined action of microbial, enzymatic and chemical reaction (acidic degradation) [41]. This type of behaviour was also found in thermal degradation of sucrose in subcritical water (high pressure and high temperature conditions) [54].

The lag phase and consequent sinusoidal behaviour observed in pure, high concentrated and neutral solutions treated at high temperatures, suggests an autocatalytic type reaction.

This autocatalytic nature is probably due to two main factors:

1. formation of weak acids during the first step of sucrose hydrolysis, that decreases solutions' $\mathrm{pH}$, thus accelerating the reaction $[70,76,90]$ and

2. molecular mobility. In the last decade, scientists started to realise the importance of molecular mobility, influenced by temperature and amount of water molecules in the system, in typical food reactions [13, 67, 69, 121]. In sucrose hydrolysis, water can act as a solvent or reactant [73]. In concentrated solutions, there is a decreased molecular mobility in the system as well as a deficit in the available water molecules for the reaction to occur, which may explain the existence of a lag phase. Furthermore, studies on sucrose crystals indicate that sucrose degradation products depress the melting point, leading to a type of autocatalysis reaction by the increased molecular mobility [91]. In the amorphous state, the increase of molecular mobility during the reaction may also be explained by lower molecular weights of the degradation products (i.e. fructose and glucose) when compared with sucrose.

Recently, these two hypothesis were confirmed: a significant effect of both temperature and water content (i.e. system's molecular mobility) on the lag time was observed [93] and a relationship between caramelisation kinetics and $\mathrm{H}^{+}$production was identified [96].

\section{Product Formation Behaviour}

Studies on kinetics of caramelisation products formation are much less common and are usually related with HMF production [7, 74]. Furthermore, such studies are carried under first-order kinetics reaction conditions, i.e. in diluted acidic solutions. However, an induction period has been observed in non-enzymatic browning of freeze dried model systems containing sucrose [68, 73], probably due to the above-mentioned low molecular mobility in these systems.

\section{Modelling the Kinetics of the Complete Caramelisation Reaction}

Due to the large number of reaction pathways and even larger number of products formed during the caramelisation reaction, kinetic studies based on the caramelisation reaction mechanism are complex. The complexity of the reaction makes the use of multiresponse modelling [17, 125] very attractive, since it also allows clarifying the main reaction pathways that directly depend on the process conditions: this modelling approach accounts for kinetic information of both reactants and products involved in the reaction, resulting in insightful parameter estimation and, consequently, in more accurate model predictions. This method was applied to the mathematical description of the sucrose caramelisation reaction [96], based on the mechanisms referred in literature (Fig. 1a). The pathway proposed in Fig. 1b fulfilled multiresponse regression analysis of the experimental data and the proposed model successfully described the experimental data (Fig. 2).

Nevertheless, it is convenient to consider that the mathematical model can only be "tentatively entertained" [18]. As an example, the determined pathway (Fig. 1b) has been described by several authors when discussing the higher reactivity of fructose over glucose $[5,7,74,76,84$, 106]. However, other pathways may emerge, if different processing conditions are used. This was observed for very low water content sucrose solutions, where the ability of the global model to predict caramelisation kinetics under such extremely low water content was impaired [96]. These conclusions are a good example of the use of mathematical models, not merely with predictive purposes, but aiming at including fundamental principles, allowing a better understanding of the complex chemical changes occurring during heating of sucrose. 
Fig. 2 Multiresponse model prediction (line) and experimental data (dots) of sucrose, fructose, glucose, HMF, furfural, 5-methylfurfural and $\mathrm{pH}$ of heat processed solutions. Example for a $25.30 \%(\mathrm{w} / \mathrm{w})$ water content sucrose solution processed at $140{ }^{\circ} \mathrm{C}[96]$
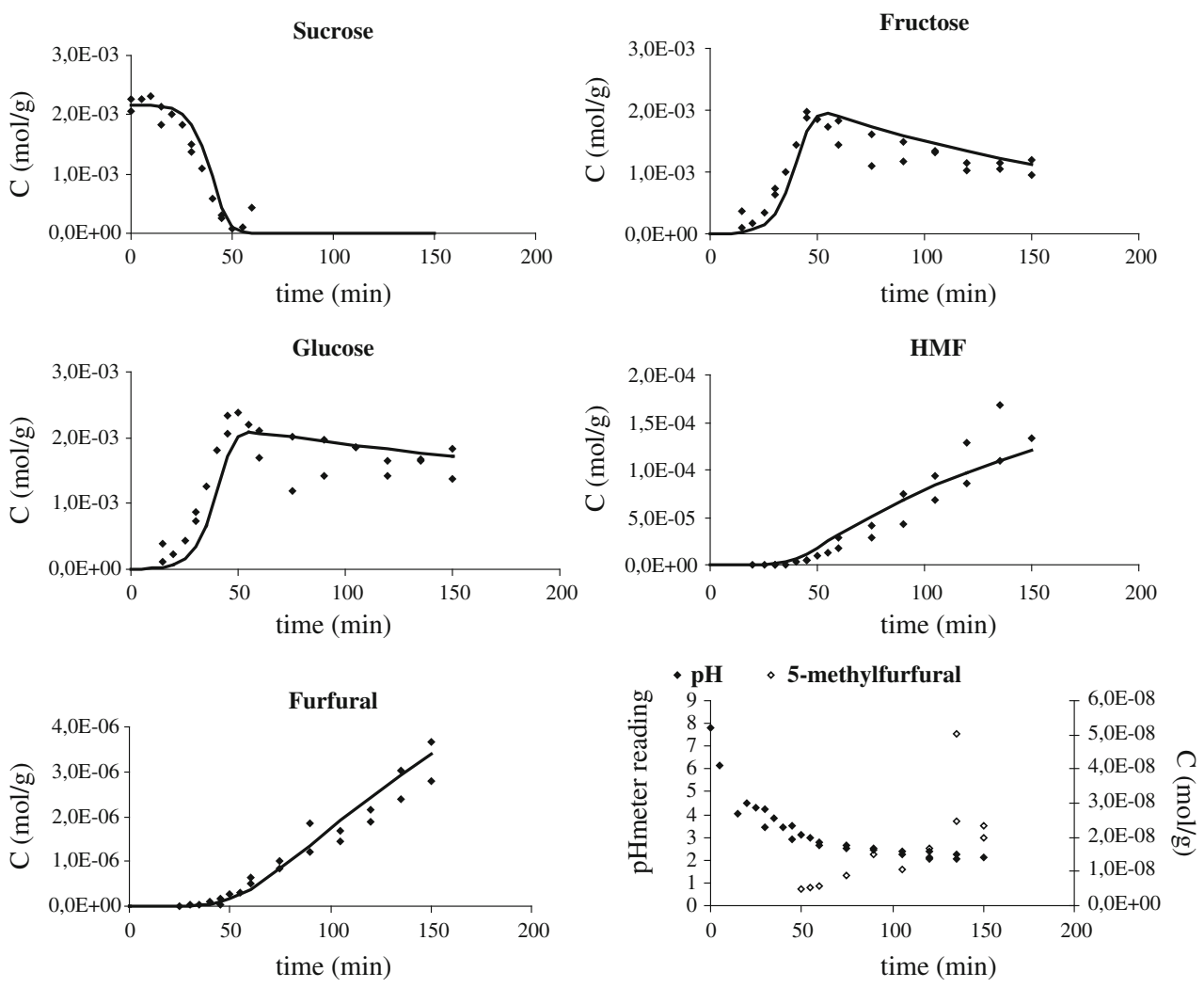

\section{Physical Behaviour of Supercooled Sucrose}

\section{Significant Phenomena in Supercooled Sucrose}

The physical state of all materials results from its composition (mainly molecular weight and water content), thermal history and temperature. These factors directly influence systems' molecular mobility, which is responsible for systems' physical properties. In food systems, molecular mobility, together with food microstructure, is also responsible for other quality attributes, like chemical reactivity and organoleptic characteristics [77].

When a liquid is cooled below the melting temperature, and crystallisation is avoided, a rubbery region or supercooled melt "state" is observed. With further cooling of the system, the "glassy state" is achieved at the glass transition temperature $\left(T_{\mathrm{g}}\right)$.

The glass transition temperature has been described as the temperature at which molecules take at least $100 \mathrm{~s}$ to move from one position to another [22]. $T_{\mathrm{g}}$ is also considered as the temperature upper limit for physical stability of a material, which supports the great importance that food scientists dedicate to this subject [121] and is dependent on both composition and solid content of a material [47]. Not without contestation [115], the "glassy state" is generally accepted to be an isoviscosity state, with viscosity $\left(\eta_{\mathrm{g}}\right)$ approximately $10^{12} \mathrm{~Pa} \mathrm{~s}$ [2], resembling more a transparent brittle solid than a free flowing liquid [107]. Glasses have also been described as non-equilibrium amorphous solids that lack long-range spatial order, typical of crystalline structures [52]. The transition from the supercooled liquid to the glassy state is not considered by some authors as a true thermodynamic transition [88] (at least in the conventional sense), since there is no observable difference in the molecule structure, but instead an increase on the relaxation time, which becomes infinite at experimental time scale. Others $[48,107]$ consider a second-order thermodynamic transition, since glass transition presents no latent heat of phase transition and a stepchange discontinuity in the heat capacity.

\section{Rheological Measurements and Viscosity in Supercooled} Sucrose Solutions

Highly concentrated sucrose solutions can be achieved by using high temperatures (above melting temperature) for short periods of time, followed by fast cooling [20, 59, 65]. This procedure leads to extremely high viscosity and amorphous solutions, with decreased molecular mobility, thus preventing crystallisation [116]. However, the material is in a metastable state: shearing promotes collision between its molecules, leading to nucleation and consequent crystal growth $[56,117]$. This metastable nature of highly concentrated sucrose solutions confines the use of 
steady-state flow measurements to characterise the rheological properties of such solutions. Due to the increasing shear involved in such experiments, crystallisation is faster and there is a change in the rheological behaviour during the measurements. This kind of phenomenon has been mentioned, when discussing the difficulty of the use of rheological methods in crystallisation studies [58].

Sucrose solutions behave as Newtonian fluids [83], and this behaviour has been reported for concentrations up to $78 \%$ (w/w) [108]. For higher sucrose concentrations, most studies mainly focus on viscosity of at temperatures below $20^{\circ} \mathrm{C}$, avoiding sample's fast crystallisation [15, 31, 85]. At room temperature $\left(20-80^{\circ} \mathrm{C}\right)$, viscosity of concentrated sugar solutions has been studied using cone and plate rheometry and the falling sphere methods [122]. However, a mixture of sucrose/fructose $(87.5 / 12.5 \%$ (w/w)) was used, expanding the sugar concentration range without crystallisation.

To asses viscosity of sucrose solutions from 70 to $85 \%$ $(\mathrm{w} / \mathrm{w})$ in a wide temperature range $\left(0-90{ }^{\circ} \mathrm{C}\right)$, avoiding crystallisation, creep test has been successfully applied [94]. Creep test can be used to assess the maximum Newtonian viscosity of a material, if time is sufficiently long to attain viscous flow. In this transient test, a small instantaneous stress is applied to the sample, and the response of strain is observed along time. After stress removal, the recovery of strain is also an indication of the rheological behaviour of the material in study [124]. The stress applied to the sample is rather low, thus minimising the disturbance in the system.

In amorphous materials such as supercooled sucrose solutions, molecular mobility is reflected on the systems viscosity, which in turn is mainly proportional to the time scale of the relaxation time. Molecular relaxation behaviour in the supercooled "state" has interested scientists for long time. Experimentally, there have been observed, mainly, two types of temperature dependence behaviour in the supercooled "region": (1) the traditionally called Arrhenius behaviour, where viscosity varies exponentially with the reciprocal of temperature, and (2) the VFT behaviour, where viscosity temperature dependence presents a non-linear behaviour in the Arrhenius plot [3]. These different behaviours have been classified as strong (Arrhenius following) and fragile (VFT following) supercooled liquids [2]. Strong liquids are usually tetrahedral network structures, with small changes in heat capacity at the glass transition temperature. On the other hand, fragile liquids do not present directional bonds, often have ionic or aromatic character and present sharp changes in heat capacity at the glass transition temperature. The fragility concept has been proposed to measure the deviation from strong behaviour in the "supercooled state" and to characterise the steepness of the temperature dependence of viscosity [3].
The concept of free volume $[34,40]$ and the entropycontrolled cooperative motions approach [1] are good examples of theories developed to describe strong/fragile relaxation behaviour. However, these theories do not consider the experimentally observed dynamical singularity in the response of a supercooled system at a critical (or divergence) temperature, $T_{\mathrm{c}}\left(T_{\mathrm{c}}>T_{\mathrm{g}}\right)$ [52].

The experimental determination of $T_{\mathrm{c}}$ using rheological measurements is difficult. The torque of most commercial rheometers is not able to support such high viscosities. In order to measure viscosity near $T_{\mathrm{g}}$, it is necessary to replace the transducer [119] or recur to comparison techniques that introduce a larger experimental error [115]. Literature values of $T_{\mathrm{g}} / T_{\mathrm{c}}$ can be found typically around 0.8 [8]: for semi-rigid polymers this ratio was found to be around 0.9 [111] and studies on glycerol indicated an approximate 0.75 ratio [115]. For sucrose solutions, $T_{\mathrm{g}} / T_{\mathrm{c}}$ was found to be approximately 0.86 , in a wide concentration range [31].

More recently, the random walk approach was used to develop a model that is able to describe relaxation temperature dependence in both strong and fragile liquids $[8,9]$. This approach has been successfully applied in supercooled sucrose solutions [95].

\section{Sucrose Crystallisation}

Sucrose crystallisation is an important phase transition in food industry and several research works have been dedicated to this subject.

Crystallisation is related with the formation of a crystalline lattice structure. Typically, this occurs in four steps: (1) generation of a supersaturated state; (2) nucleation, i.e. the formation of the lattice structure; (3) growth, i.e. subsequent growth of nuclei until equilibrium is attained; and (4) recrystallisation, i.e. reorganisation of the crystalline structure to a lower energy state [58]. In highly concentrated sucrose solutions and at room temperature and higher, crystallisation phenomenon, as well as its kinetic behaviour, temperature and water content dependence, have been largely discussed $[56,58,59,117]$.

\section{Mathematical Modelling in the Supercooled State}

In the area of quality/safety loss kinetics during storage and processing, some remarkable examples of the effort made to draw food scientists' attention to the benefits of accurate modelling and reporting of experimental data are available (e.g. $[6,72,78,109])$. Later works $[33,131]$ also played a critical role on alerting to the importance of estimates precision and quality of the regression. However, regression analysis is less common in works describing molecular relaxations in food products. Many studies in this area fit models with "universal constant" parameters (e.g. [122]) 
and extrapolate such "description" to "predict" relaxation outside the experimental range (e.g. [21]). Moreover, studies on estimates precision are extremely rare [87].

\section{Empirical Modelling of Molecular Relaxation}

Temperature Dependence Following Arrhenius Type Behaviour Viscosity dependence on temperature for Newtonian fluids is extensively described using an Arrhenius type equation [12]. This is an empirical model, developed from the theory of the liquid state, based on the movement of molecules through the formation of "holes" in the system [44]. The Arrhenius model has been successfully applied to describe the viscosity temperature dependence of various food products, such as clarified fruit juices [51, 60,62, 134], vegetable oils in solution [63] and molasses [128]. For sucrose solutions, this type of behaviour has been reported for low concentrations (up to $60 \%$ ) -i.e. solutions with real "liquid like" behaviour [93].

Temperature Dependence Deviating from Arrhenius Behaviour At temperatures near the glass transition, more specifically in the $T_{\mathrm{g}}$ to $T_{\mathrm{g}}+100{ }^{\circ} \mathrm{C}$ range, experimental data of fragile glass-forming liquids show markedly deviation from Arrhenius behaviour. This behaviour has been described by several mathematical functions (e.g. the Vogel-Fulcher-Tammann (VFT) equation [2]). However, food scientists have shown a preference for using the Williams, Landel and Ferry (WLF) empirical model to describe the viscosity temperature dependence (Eq. 1) in the above-mentioned temperature range $[47,136]$ :

$\log \eta=\log \eta_{\mathrm{ref}}-\frac{c_{1}\left(T-T_{\mathrm{ref}}\right)}{c_{2}+T-T_{\mathrm{ref}}}$

The constants $c_{1}$ and $c_{2}$ became "universal", respectively, with the values of 17.44 and $51.6{ }^{\circ} \mathrm{C}$, using $T_{\mathrm{g}}$ as reference temperature.

The WLF model has been used to describe the viscosity temperature dependence of several food materials, mainly at sub ambient temperatures, such as amorphous isomalt [104], sucrose solutions [15, 31, 85] and solutions of other low molecular weight carbohydrates, such as glucose, fructose, sorbitol, xylitol [79] and trehalose.

The use of the "universal" constants $c_{1}$ and $c_{2}$ is limited, and Williams, Landel and Ferry themselves cautioned for the indiscriminate use of $T_{\mathrm{g}}$ as reference temperature, since small differences in measured $T_{\mathrm{g}}$ could lead to a deviation in the curve behaviour [136]. Other authors also questioned the universality of these constants, when developing a molecular-kinetic theory to explain the temperature dependence of relaxation behaviour in glass-forming liquids [1]. In this study, a similar expression to the WLF model (Eq. 1) was found. However, $c_{1}$ was dependent on

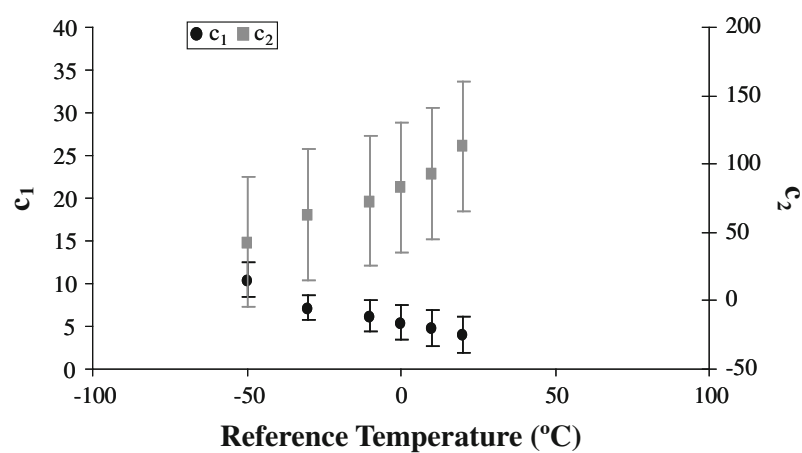

Fig. 3 Reference temperature effect on the WLF $c_{1}$ and $c_{2}$ parameters $(79.93 \%(\mathrm{w} / \mathrm{w})$ sucrose concentration). The bars indicate the $95 \%$ confidence interval limits [94]

$T_{\text {ref }}$ and on the nature of the material itself, and $c_{2}$ dependent on both $T_{\text {ref }}$ and process temperature $(T)$.

The use of universal $c_{1}$ and $c_{2}$ constants to describe relaxation behaviour in supercooled systems was also questioned by other researchers, which found GordonTaylor type concentration dependencies for both $c_{1}$ and $c_{2}$ [20]. In polymers, several works have also reported $c_{2}$ "constants" far from universal [4]. More recently, another work demonstrated that a better prediction of the viscosity is achieved if the reference temperature, used in the WLF model, is within the experimental data range, and $c_{1}$ and $c_{2}$ are model parameters [87]. This approach has been successfully applied in honeys [123].

For supersaturated sucrose solutions the WLF model successfully described the observed behaviour [94]. Moreover, a minimum value for the estimates standard error ( $\log \eta_{\mathrm{ref}}, c_{1}$ and $c_{2}$ ) was observed when the reference temperature was close to the lowest temperature used in the experimental determination of viscosity (example in Fig. 3) and this is in accordance with Peleg [87]. In addition, it was observed that when the reference temperature is fixed, $c_{1}$ is dependent on the concentration, whereas $c_{2}$ does not present statistically significant concentration dependence (example in Fig. 4). These findings were in agreement with Adam and Gibbs [1] conclusions.

\section{The Random Walk Approach to Modelling Molecular Relaxation}

As already discussed, Arkipov and Bassler's proposed a random walk (RW) approach for describing molecular relaxation in supercooled liquids. A random walk is a statistical tool that can be used to predict the final state of a system when a physical phenomenon, usually associated with movement, occurs in a complex way. It is constituted by steps of the same length and, at each point, the probability of displacement into another point is independent of the actual position and equal for all new probable locations. 


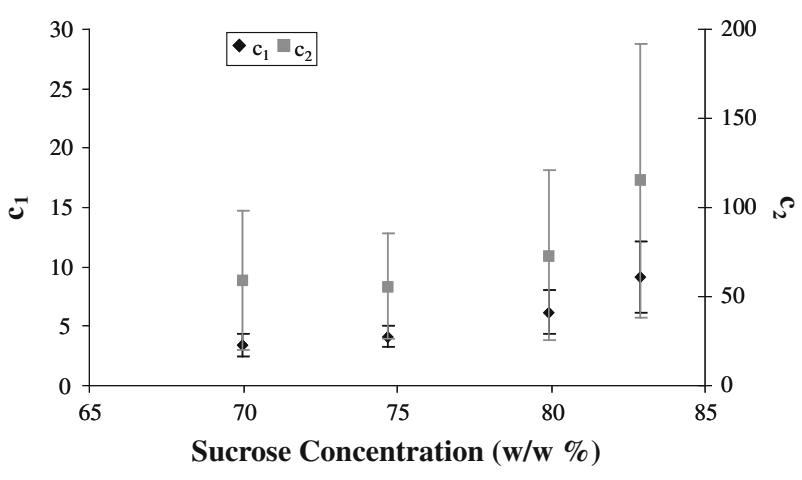

Fig. 4 Concentration dependence of the WLF $c_{1}$ and $c_{2}$ parameters, using $0{ }^{\circ} \mathrm{C}$ as reference temperature. The bars indicate the $95 \%$ confidence interval limits [94]

This approach has been successfully applied to semirigid polymers [111] and it is based on a random walk modelling of molecular movements in the system. To some extent, it is possible to affirm that this is a model based on "movement mechanism" and a parallelism can be established with the mechanistic modelling of chemical reactions kinetics. Herein, a small summary of this modelling approach is presented.

Arkipov and Bassler's random walk approach [8, 9] considers that a unit structure (i.e. a molecule or a subunit embedded in the molecular environment) in the configurational space, due to existing strong interactions, cannot change its position without the adjacent structures movement and, consequently, a transition in the configurational space occurs. This transition can be considered as a jump of a unit structure in a highly complex energy landscape. After each jump, the structural unit finds a new environment and the configurational space phase memory is lost. This "no memory" process can be modelled as a random walk on a disordered network of hopping sites in configurational space, characterised by a broad distribution of energies. Considering that the energy of a structural unit depends on a large number of configurational co-ordinates, each one varying randomly, a normalised function can be realistically assumed for the distribution of possible metastable states. Arkipov and Bassler defined the random variable $(E)$ as the energy to supply to a structural unit for the unit to "jump" to another site in the configurational space. Considering a random walk, the jump frequency of structural units can be determined using a master equation for the normalised energy distribution function of the structural units [8].

Being the viscosity proportional to the inverse of the jump frequency, the temperature dependence of viscosity for fragile glass-forming liquids in the higher temperature regime (or "real liquid", where the energy landscape is fluctuating at a frequency large enough to allow an unit jump to an adjacent site, and the activation energy of a jump upward in energy is the difference between sites energies $\left.\left(T>T_{\mathrm{c}}\right)\right)$ is then given by $[8,9]$ :

$$
\begin{aligned}
\eta= & \eta_{0_{\mathrm{RW}}} 2^{(2 \beta-1) / 2(\beta-1)} \frac{1}{\beta} \Gamma\left(\frac{1}{\beta}\right)\left(\frac{T_{\mathrm{O}_{\mathrm{RW}}}}{T}\right) \\
& \times \exp \left[(\beta-1)\left(1-2^{-1 /(\beta-1)}\right)\left(\frac{T_{0_{\mathrm{RW}}}}{\beta T}\right)^{\beta /(\beta-1)}\right]
\end{aligned}
$$

where $\beta$ is a shape parameter of the normalised distribution of the random variable $(E), \Gamma$ is the gamma function, $\eta_{0_{\mathrm{RW}}}$ accounts for the contribution of structural unit jumps towards viscosity and $T_{0_{\mathrm{RW}}}$ is a temperature that reflects the spread of the self-energies of the structural units.

This equation was successfully applied for describing the temperature dependence of supercooled sucrose solutions viscosity (Fig. 5). The obtained results are relevant for the physical interpretation of the observed phenomena (Table 1): (1) $\eta_{0_{\mathrm{RW}}}$, which is proportional to the structural units jumps contribution to viscosity, increased with sucrose content (i.e. with number of sucrose molecules in the solution) and (2) the spread of the self-energies of the structural units due to configurational disorder, which is described by $T_{0_{\mathrm{RW}}}$, decreased with increasing sucrose content [95].

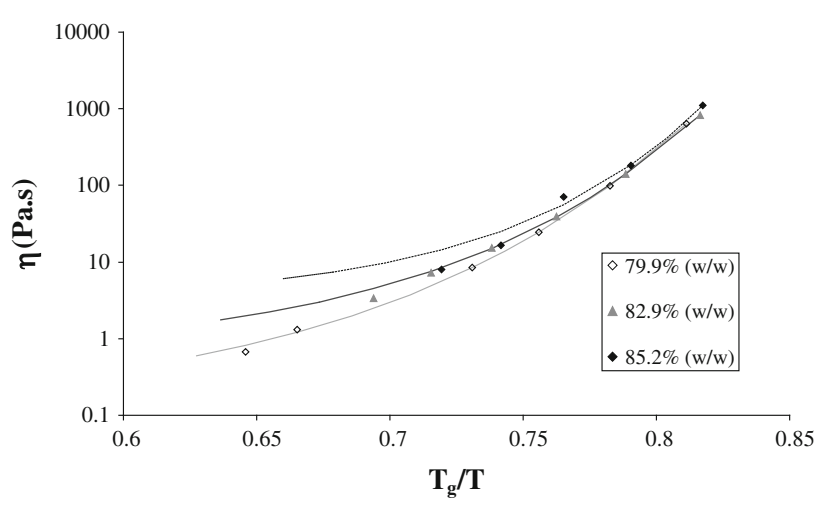

Fig. 5 Random walk model fitting to experimental data of viscosity for three sucrose concentration levels: $79.9 \%(\mathrm{w} / \mathrm{w})$, solid grey line; $82.9 \%(\mathrm{w} / \mathrm{w})$, solid black line and $85.2 \%(\mathrm{w} / \mathrm{w})$, dashed line [95]

Table 1 Results from fitting the random walk (RW) model to experimental data, at different sucrose concentrations [95]

\begin{tabular}{lllll}
\hline $\begin{array}{l}\text { Sucrose } \\
\text { concentration } \\
(\mathrm{w} / \mathrm{w})\end{array}$ & \multicolumn{4}{l}{ Estimated parameters } \\
\cline { 2 - 5 } & $\eta_{0_{\mathrm{RW}}}$ & $T_{0_{\mathrm{RW}}}$ & $\beta$ & $m_{\mathrm{RW}}$ \\
\hline 79.9 & $6.88 \times 10^{-3}$ & 541.64 & 1.16 & 118.61 \\
82.9 & $2.13 \times 10^{-2}$ & 502.05 & 1.13 & 151.46 \\
85.2 & $4.03 \times 10^{-2}$ & 455.23 & 1.09 & 285.46 \\
\hline
\end{tabular}




\section{Assessing Fragility of a Glass-Forming Liquid}

'Fragility' is a term that tries to capture the deviation of Arrhenius behaviour in the "supercooled state". Several methods have been proposed to "measure" fragility of a system, i.e. to characterise the steepness of the temperature dependence of viscosity [3]. The most popular form is to measure the slope $(\mathrm{m})$ of the VFT (or WLF) equation near $T_{\mathrm{g}}[3,21,89,112,115]$ :

The RW model also allows the description of the function steepness through the $\beta$ parameter. In fact, strong glass-forming fluids behaviour (Arrhenius-like) show $\beta \rightarrow 2$ and fragile glass-forming liquids behaviour (VFTlike) indicates $\beta \rightarrow 1$ [9]. Furthermore, a correlation of the fragility index $\beta$ and $\mathrm{m}$ has been successfully established [110]:

$m_{\mathrm{RW}}=\frac{1}{\ln 10}\left[\left(\frac{2-\beta}{2(\beta-1)}\right)+\beta\left(\frac{T_{0_{\mathrm{RW}}}}{\beta T_{\mathrm{g}}}\right)^{\beta /(\beta-1)}\right]$

In supercooled sucrose solutions, the fragility index $(\beta)$ was found to be significantly affected by solution concentration (Table 1; [95] and approached 1 with increasing sucrose content as reported in literature [4, 9]. Furthermore, it was possible to determine an $m_{\mathrm{RW}}$ (from Eq. 3) in agreement with literature values of sucrose solutions fragility [21].These reported results are even more significant when the small concentration range of the study is considered, and are a good indication of the potential of the random walk (RW) approach to the study of molecular relaxation behaviour in the supercooled "state", using only high temperature regime data.

Academically, this study presents a great potential for investigating reactions and phase transitions in such systems. Application of such knowledge may help, in the future, the development of stable food products with improved functionality.

\section{Summary and Future Research Trends in Supercooled Sucrose Solutions}

This paper reviewed critical issues and research on highly concentrated sucrose solutions. Concerning sucrose thermal degradation in such solutions, two main topics were addressed: (1) autocatalytic behaviour of degradation kinetics (2) and description of the caramelisation reaction, i.e. sucrose degradation and product formation. As for the relaxation behaviour of the supercooled sucrose solutions, main topics are: (1) the rheological properties of highly concentrated sucrose solutions at ambient temperatures, (2) currently used theories of the viscosity temperature dependence with special emphasis on a critical assessment of the use of $c_{1}$ and $c_{2}$ "universal" constants and $T_{\mathrm{g}}$ as reference temperature, to describe viscosity temperature dependence in WLF type behaviour and (3) the random walk approach on description of sucrose relaxation behaviour in the supercooled "state".

The reviewed works allow identifying several issues with great potential for contributing to significant advances in Food Science and Technology.

Greater insight into the mechanism of sucrose thermal degradation can be gained by performing experiments measuring other reaction products, e.g. the organic acids. The study of the reaction mechanism under different processing conditions (e.g. high/extremely low water content and different initial $\mathrm{pH}$ ) may also reveal different reaction pathways. The kinetic modelling procedures here presented can be enhanced by studies on improving parameters and predictions precision by optimal experimental design. Special relevance should be given to multiresponse regression procedures, where optimal experimental designs were not yet applied in the food science area.

Studies on the effect of sucrose degradation on viscosity of solutions and its effect on temperature and water content behaviour may contribute to a better understanding of the relationship between composition and physical behaviour. Such understanding is a potential key area in food studies in the next years.

The random walk approach to molecular mobility of supercooled systems may prove to be useful to study and predict other processes. The random nature of molecular collision during crystallisation makes such phenomena of great interest to observe using a random walk approach. The diffusion in supercooled and glassy systems may also be studied using this approach-such studies may be extremely useful in understanding and predicting important properties of a food product, like, for example, controlled release of functional ingredients in the digestible track.

Acknowledgments Authors are grateful for the valuable discussions with Teresa S. Brandão and Rosiane Lopes da Cunha during this research. Author M. A. C. Quintas acknowledges the financial support of her research by FCT grant SFRH/BPD/41715/2007.

\section{References}

1. Adam G, Gibbs JH (1965) On the temperature dependence of cooperative relaxation properties in glass-forming liquids. J Chem Phys 43(1):139-146

2. Angell CA (1988) Perspective on the glass-transition. J Phys Chem Solids 49(8):863-871

3. Angell CA (2002) Liquid fragility and the glass transition in water and aqueous solutions. Chem Rev 102(8):2627-2649

4. Angell CA, Bressel RD, Green JL, Kanno H, Oguni M, Sare EJ (1994) Liquid fragility and the glass-transition in water and aqueous-solutions. J Food Eng 22(1-4):115-142 
5. Antal MJ Jr, Mok WSL, Richards GN (1990) Mechanism of formation of 5-(hydroxymethyl)-2-furaldehyde from D-fructose and sucrose. Carbohydr Res 199(1):91-109

6. Arabshahi A, Lund D (1985) Considerations in calculating kinetic parameters from experimental data. J Food Process Eng 7(4):239-251

7. Arena E, Fallico B, Maccarone E (2001) Thermal damage in blood orange juice: kinetics of 5-hydroxymethyl-2-furancarboxaldehyde formation. Int J Food Sci Technol 36(2):145-151

8. Arkhipov VI, Bassler H (1994) Random-walk approach to dynamic and thermodynamic properties of supercooled melts. 1 . Viscosity and average relaxation-times in strong and fragile liquids. J Phys Chem 98(2):662-669

9. Arkhipov VI, Bassler H (1994) A random-walk model of relaxations in supercooled melts. J Non-Cryst Solids 172: 396-400

10. Baek YJ, Cho YH, Huh CS, Lee JJ, Park IB, Park JY (2005) Encapsulation of egg yolk immunoglobulins and use thereof. Korea Yakult Co Ltd. Patent Number(s): KR2004071109-A

11. Baisier WM, Labuza TP (1992) Maillard browning kinetics in a liquid model system. J Agric Food Chem 40(5):707-713

12. Barnes HA, Hutton JF, Walters K (1989) An introduction to rheology, 1st edn. Elsevier Science Publishers B.V., Amsterdam

13. Bell LN, Labuza TP (1994) Influence of the low-moisture state on $\mathrm{pH}$ and its implication for reaction kinetics. J Food Eng 22(1-4):291-312

14. Bell LN, White KL, Chen YH (1998) Maillard reaction in glassy low-moisture solids as affected by buffer type and concentration. J Food Sci 63(5):785-788

15. Bellows RJ, King CJ (1973) Product collapse during freezedrying of liquid foods. AICHE Symp Ser 69(132):33-41

16. BeMiller JN, Whistler RL (1996) Carbohydrates. In: Fennema OR (ed) Food chemistry, 3rd edn. Marcel Dekker, Inc., New York, pp 157-224

17. Box GEP, Draper NR (1965) The Bayesian estimation of common parameters from several responses. Biometrika 52 (3-4):355-365

18. Box GEP, Draper NR (1965) The experimental study of physical mechanisms. Technometrics 7(1):23-42

19. Bozkurt H, Gogus F, Eren S (1999) Nonenzymic browning reactions in boiled grape juice and its models during storage. Food Chem 64(1):89-93

20. Braga da Cruz I, MacInnes WM, Oliveira JC, Malcata FX (2002) Supplemented state diagram for sucrose from dynamic mechanical thermal analysis. In: Levine $\mathrm{H}$ (ed) Amorphous food and pharmaceutical systems. The Royal Society of Chemistry, Cambridge, pp 59-70

21. Branca C, Magazu S, Maisano G, Migliardo F, Migliardo P, Romeo G (2001) Alpha, alpha-trehalose/water solutions. 5. Hydration and viscosity in dilute and semidilute disaccharide solutions. J Phys Chem B 105(41):10140-10145

22. Bueche $F$ (1959) Mobility of molecules in liquids near the glass temperature. J Chem Phys 30(3):748-752

23. Buera MD, Chirife J, Resnik SL, Lozano RD (1987) Nonenzymatic browning in liquid model systems of high water activitykinetics of color changes due to caramelization of various single sugars. J Food Sci 52(4):1059-1062

24. Buera MD, Chirife J, Resnik SL, Lozano RD (1987) Nonenzymatic browning in liquid model systems of high water activitykinetics of color changes due to reaction between glucose and glycine peptides. J Food Sci 52(4):1068-1070

25. Buera MD, Chirife J, Resnik SL, Wetzler G (1987) Nonenzymatic browning in liquid model systems of high water activitykinetics of color changes due to Maillard reaction between different single sugars and glycine and comparison with caramelization browning. J Food Sci 52(4):1063-1067
26. Burdurlu HS, Karadeniz F (2003) Effect of storage on nonenzymatic browning of apple juice concentrates. Food Chem 80(1): 91-97

27. Cais-Sokolinska D, Pikul J, Dankow R, Wojtowski J (2005) Changes in some selected physico-chemical and sensory parameters of UHT milk during storage at different temperatures. Milchwissenschaft 60(1):37-40

28. Carabasa-Giribet M, Ibarz-Ribas A (2000) Kinetics of colour development in aqueous fructose systems at high temperatures. J Sci Food Agric 80(14):2105-2113

29. Carabasa-Giribet M, Ibarz-Ribas A (2000) Kinetics of colour development in aqueous glucose systems at high temperatures. J Food Eng 44(3):181-189

30. Carabasa M, Ibarz A, Garza S, Barbosa-Canovas GV (1998) Removal of dark compounds from clarified fruit juices by adsorption processes. J Food Eng 37(1):25-41

31. Champion D, Hervet H, Blond G, Le Meste M, Simatos D (1997) Translational diffusion in sucrose solutions in the vicinity of their glass transition temperature. J Phys Chem B 101(50): 10674-10679

32. Clarke MA, Edye LA, Eggleston G (1997) Sucrose decomposition in aqueous solution, and losses in sugar manufacture and refining. Adv Carbohydr Chem Biochem 52:441-470

33. Cohen E, Birk Y, Mannheim CH, Saguy IS (1994) Kinetic parameter-estimation for quality change during continuous thermal-processing of grapefruit juice. J Food Sci 59(1):155158

34. Cohen MH, Turnbull D (1959) Molecular transport in liquids and glasses. J Chem Phys 31(5):1164-1169

35. Cuq B, Gontard N, Guilbert S (1997) Thermoplastic properties of fish myofibrillar proteins: application to biopackaging fabrication. Polymer 38(16):4071-4078

36. Davies C, Labuza T (1997) Maillard browning of confectioneries. In: Ziegler G (ed) Confectionery science, 1st edn. Penn State Press, Happy Valley, pp 35-66

37. de Bruijn JM, Struijs JLM, Bout-Diederen MEF (1999) Sucrose degradation and color formation. Zuckerindustrie 124(1):28-33

38. Defaye J, Garcia Fernandez JM (1994) Protonic and thermal activation of sucrose and the oligosaccharide composition of caramel. Carbohydr Res 256(2):C1-C4

39. Defaye J, Garcia Fernandez JM (1995) The oligosaccharide components of caramel. Zuckerindustrie 120(8):700-704

40. Doolittle AK (1951) Studies in Newtonian flow. II. The dependence of the viscosity of liquids on free-space. J Appl Phys 22 (12):1471-1475

41. Eggleston G (2002) Deterioration of cane juice-sources and indicators. Food Chem 78(1):95-103

42. Eggleston G, Trask-Morrel B, Vercellotti JR (1996) Use of differential scanning calorimetry and thermogravimetric analysis to characterize the thermal degradation of crystalline sucrose and dried sucrose-salt residues. J Agric Food Chem 44(10): 3319-3325

43. Eggleston G, Vercellotti JR (2000) Degradation of sucrose, glucose and fructose in concentrated aqueous solutions under constant $\mathrm{pH}$ conditions at elevated temperature. J Carbohydr Chem 19(9):1305-1318

44. Eyring H, Hirschefelder J (1937) The theory of the liquid state. J Phys Chem 41(2):249-257

45. Fallico B, Arena E, Zappala M (2003) Roasting of hazelnuts. Role of oil in colour development and hydroxymethylfurfural formation. Food Chem 81(4):569-573

46. Fernandez-Artigas P, Guerra-Hernandez E, Garcia-Villanova B (1999) Browning indicators in model systems and baby cereals. J Agric Food Chem 47(7):2872-2878

47. Ferry JD (1980) Viscoelastic properties of polymers, 3rd edn. Wiley, New York 
48. Fox TG, Flory PJ (1950) 2nd-order transition temperatures and related properties of polystyrene. 1. Influence of molecular weight. J Appl Phys 21(6):581-591

49. Fundo J, Quintas MAC, Brandão TRS, Silva CLM (2009) Development of a safer formulation of egg yolk pudding: functional and sensory assessment. J Food Process Preserv. doi: 10.1111/j.1745-4549.2009.00472.x

50. Garza S, Ibarz A, Pagan J, Giner J (1999) Non-enzymatic browning in peach puree during heating. Food Res Int 32(5): 335-343

51. Giner J, Ibarz A, Garza S, Xhian-Quan S (1996) Rheology of clarified cherry juices. J Food Eng 30(1-2):147-154

52. Gotze W, Sjorgen L (1992) Relaxation processes in supercooled liquids. Rep Prog Phys 55(3):241-376

53. Guerra-Hernandez E, Leon C, Corzo N, Garcia-Villanova B, Romera JM (2002) Chemical changes in powdered infant formulas during storage. Int J Dairy Technol 55(4):171-176

54. Haghighat-Khajavi S, Kimura Y, Oomori T, Matsuno R, Adachi S (2005) Kinetics on sucrose decomposition in subcritical water. LWT Food Sci Technol 38(3):297-302

55. Hagiwara T, Hartel RW (1996) Effect of sweetener, stabilizer, and storage temperature on ice recrystallization in ice cream. J Dairy Sci 79(5):735-744

56. Hartel RW (1993) Controlling sugar crystallization in food products. Food Technol 47(11):99-106

57. Hartel RW (1996) Ice crystallization during the manufacture of ice cream. Trends Food Sci Technol 7(10):315-321

58. Hartel RW (2001) Crystallization in foods, 1st edn. Aspen Publishers, Inc., Gaithersburg

59. Howell TA Jr, Ben-Yoseph E, Rao C, Hartel RW (2002) Sucrose crystallization kinetics in thin films at elevated temperatures and supersaturations. Cryst Growth Des 2(1):67-72

60. Ibarz A, Gonzalez C, Esplugas S (1994) Rheology of clarified fruit juices. III: orange juices. J Food Eng 21(4):485-494

61. Ibarz A, Pagan J, Garza S (2000) Kinetic models of non-enzymatic browning in apple puree. J Sci Food Agric 80(8):11621168

62. Ibarz A, Pagan J, Miguelsanz R (1992) Rheology of clarified fruit juices. II: blackcurrant juices. J Food Eng 15(1):63-73

63. Igwe IO (2004) The effects of temperature on the viscosity of vegetable oils in solution. Ind Crop Prod 19(2):185-190

64. Imming R, Buczys R, Lehnberger A, Bliesener KM (1996) A new approach to the kinetics of colour formation in concentrated carbohydrate solutions. Starch Starke 48(5):163-166

65. Izzard MJ, Ablett S, Lillford PJ (1991) Calorimetric study of the glass transition occurring in sucrose solutions. In: Dickinson E (ed) Food polymers, gels and colloids. The Royal Society of Chemistry, Cambridge, pp 289-309

66. Kadakal C, Nas S (2003) Effect of heat treatment and evaporation on patulin and some other properties of apple juice. J Sci Food Agric 83(9):987-990

67. Karel M (1993) Temperature-dependence of food deterioration processes. J Food Sci 58(6):ii

68. Karel M, Labuza TP (1968) Nonenzymatic browning in model systems containing sucrose. J Agric Food Chem 16(5):717-719

69. Kouassi K, Roos YH (2001) Glass transition and water effects on sucrose inversion in noncrystalline carbohydrate food systems. Food Res Int 34(10):895-901

70. Kroh LW (1994) Caramelisation in food and beverages. Food Chem 51(4):373-379

71. Kumar P, Mishra HN (2004) Storage stability of mango soy fortified yoghurt powder in two different packaging materials: HDPP and ALP. J Food Eng 65(4):569-576

72. Labuza TP, Kamman JF (1983) Reaction kinetics and accelerated tests simulation as function of temperature. In: Saguy I (ed)
Computer aided techniques in food technology. Marcel Dekker, Inc., New York

73. Labuza TP, Tannenbaum SR, Karel M (1970) Water content and stability of low moisture and intermediate-moisture foods. Food Technol 24(5):543-548

74. Lee HS, Nagy S (1988) Relationship of sugar degradation to detrimental changes in citrus juice quality. Food Technol 42(10):91-97

75. Lievonen SM, Laaksonen TJ, Roos YH (1998) Glass transition and reaction rates: nonenzymatic browning in glassy and liquid systems. J Agric Food Chem 46(7):2778-2784

76. Lowary TL, Richards GN (1988) Effects of impurities on hydrolysis of sucrose in concentrated aqueous solutions. Int Sugar J 90(1077):164-167

77. Ludescher RD, Shah NK, McCaul CP, Simon KV (2001) Beyond $T_{\mathrm{g}}$ : optical luminescence measurements of molecular mobility in amorphous solid foods. Food Hydrocolloids 15(4-6): 331-339

78. Lund DB (1983) Considerations in modeling food processes. Food Technol 37(1):92-94

79. Maltini E, Anese M (1995) Evaluation of viscosities of amorphous phases in partially frozen systems by WLF kinetics and glass transition temperatures. Food Res Int 28(4):367-372

80. Manley-Harris M, Richards GN (1991) Formation of trisaccharides (kestoses) by pyrolysis of sucrose. Carbohydr Res 219:101-113

81. Manley-Harris M, Richards GN (1996) Di-D-fructose dianhydrides and related oligomers from thermal treatments of inulin and sucrose. Carbohydr Res 287(2):183-202

82. Manley-Harris M, Richards GN (1997) Dihexulose dianhydrides. Adv Carbohydr Chem Biochem 52:207-266

83. Mathlouthi M, Génotelle J (1995) Rheological properties of sucrose solutions and suspensions. In: Mathlouthi M, Reiser P (eds) Sucrose properties and applications. Blackie Academic \& Professional, London, pp 126-154

84. Mauch W (1971) Chemical properties of sucrose. Sugar Technol Rev 1:239-290

85. Parker R, Ring SG (1995) A theoretical analysis of diffusioncontrolled reactions in frozen solutions. Cryo Letters 16: 197-208

86. Patel AA, Gandhi H, Singh S, Patil GR (1996) Shelf-life modeling of sweetened condensed milk based on kinetics of Maillard browning. J Food Process Preserv 20(6):431-451

87. Peleg M (1992) On the use of the WLF model in polymers and foods. Crit Rev Food Sci Nutr 32(1):59-66

88. Perez J (1994) Theories of liquid-glass transition. J Food Eng 22(1-4):89-114

89. Plazek DJ, Ngai KL (1991) Correlation of polymer segmental chain dynamics with temperature-dependent time-scale shifts. Macromolecules 24(5):1222-1224

90. Poncini L (1980) Thermal degradation of sucrose in solution-a review. Int Sugar J 82(983):332-335

91. Poncini L (1981) Thermal degradation of sucrose in the crystalline phase and melt. A review. La Sucrerie Belge 100:221228

92. Quintas M (2007) Fundamental studies on sugar syrups towards the development of the traditional confectionery industry: rheological behaviour, caramelisation and modelling. Unpublished $\mathrm{PhD}$, Catholic University of Portugal, Porto

93. Quintas M, Brandao TRS, Silva CLM (2007) Modelling autocatalytic behaviour of a food model system-sucrose thermal degradation at high concentrations. J Food Eng 78(2):537-545

94. Quintas M, Brandao TRS, Silva CLM, Cunha RL (2006) Rheology of supersaturated sucrose solutions. J Food Eng 77(4): $844-852$ 
95. Quintas M, Brandao TRS, Silva CLM, Cunha RL (2007) Modelling viscosity temperature dependence of supercooled sucrose solutions - the random-walk approach. J Phys Chem B 111(12):3192-3196

96. Quintas M, Guimaraes C, Baylina J, Brandao TRS, Silva CLM (2007) Multiresponse modelling of the caramelisation reaction. Innov Food Sci Emerg Technol 8(2):306-315

97. Quintas MAC, Brandao TRS, Silva CLM (2007) Modelling colour changes during the caramelisation reaction. J Food Eng 83(4):483-491

98. Ramirez-Jimenez A, Garcia-Villanova B, Guerra-Hernandez E (2000) Hydroxymethylfurfural and methylfurfural content of selected bakery products. Food Res Int 33(10):833-838

99. Ramirez-Jimenez A, Garcia-Villanova B, Guerra-Hernandez E (2001) Effect of toasting time on the browning of sliced bread. J Sci Food Agric 81(5):513-518

100. Ramirez-Jimenez A, Guerra-Hernandez E, Garcia-Villanova B (2000) Browning indicators in bread. J Agric Food Chem 48(9):4176-4181

101. Ramirez-Jimenez A, Guerra-Hernandez E, Garcia-Villanova B (2003) Evolution of non-enzymatic browning during storage of infant rice cereal. Food Chem 83(2):219-225

102. Ratsimba V, Fernandez JMG, Defaye J, Nigay H, Voilley A (1999) Qualitative and quantitative evaluation of mono- and disaccharides in D-fructose, D-glucose and sucrose caramels by gas-liquid chromatography-mass spectrometry: di-D-fructose dianhydrides as tracers of caramel authenticity. J Chromatogr A 844(1-2):283-293

103. Rattanathanalerk M, Chiewchan N, Srichumpoung W (2005) Effect of thermal processing on the quality loss of pineapple juice. J Food Eng 66(2):259-265

104. Raudonus J, Bernard J, Jan[ss]en H, Kowalczyk J, Carle R (2000) Effect of oligomeric or polymeric additives on glass transition, viscosity and crystallization of amorphous isomalt. Food Res Int 33(1):41-51

105. Reyes FGR, Poocharoen B, Wrolstad RE (1982) Maillard browning reaction of sugar-glycine model systems - changes in sugar concentration, color and appearance. J Food Sci 47(4): $1376-1377$

106. Richards GN (1986) Initial steps in thermal degradation of sucrose. Int Sugar J 88(1052):145-148

107. Roos YH (1995) Phase transitions in foods. Academic Press, San Diego

108. Saggin R, Coupland JN (2004) Rheology of xanthan/sucrose mixtures at ultrasonic frequencies. J Food Eng 65(1):49-53

109. Saguy I, Karel M (1980) Modeling of quality deterioration during food processing and storage. Food Technol 34(2):78-85

110. Saiter A, Bureau E, Zapolsky H, Marais S, Saiter JM (2002) Application of random walk model to the glass transition of unsaturated polyester resins cured with different styrene contents. J Non-Cryst Solids 307:738-743

111. Saiter A, Bureau E, Zapolsky H, Saiter JM (2004) Cooperativity range and fragility in vitreous polymers. J Non-Cryst Solids 345-46:556-561

112. Saiter A, Saiter JM, Grenet J (2006) Cooperative rearranging regions in polymeric materials: relationship with the fragility of glass-forming liquids. Eur Polym J 42(1):213-219

113. Schebor C, Buera MD, Karel M, Chirife J (1999) Color formation due to non-enzymatic browning in amorphous, glassy, anhydrous, model systems. Food Chem 65(4):427-432

114. Schoebel T, Tannenbaum SR, Labuza TP (1969) Reaction at limited water concentration-1. Sucrose hydrolysis. J Food Sci 34(4):324-329
115. Schroter K, Donth E (2000) Viscosity and shear response at the dynamic glass transition of glycerol. J Chem Phys 113(20): 9101-9108

116. Seo JA, Oh JY, Kim DJ, Kim HK, Hwang YH (2004) Making monosaccharide and disaccharide sugar glasses by using microwave oven. J Non-Cryst Solids 333(1):111-114

117. Shastry AV, Hartel RW (1996) Crystallization during drying of thin sucrose films. J Food Eng 30(1-2):75-94

118. Shen SC, Wu JSB (2004) Maillard browning in ethanolic solution. J Food Sci 69(4):C273-C279

119. Shi XF, Mandanici A, McKenna GB (2005) Shear stress relaxation and physical aging study on simple glass-forming materials. J Chem Phys 123(17):174507

120. Singh KJ, Roos YH (2006) State transitions and freeze concentration in trehalose-protein-cornstarch mixtures. LWT Food Sci Technol 39(8):930-938

121. Slade L, Levine H (1991) Beyond water activity: recent advances based on an alternative approach to the assessment of food quality and safety. Crit Rev Food Sci Nutr 30(2-3):115360

122. Soesanto T, Williams MC (1981) Volumetric interpretation of viscosity for concentrated and dilute sugar solutions. J Phys Chem 85(22):3338-3341

123. Sopade PA, Halley P, Bhandari B, D'Arcy B, Doebler C, Caffin N (2003) Application of the Williams-Landel-Ferry model to the viscosity-temperature relationship of Australian honeys. J Food Eng 56(1):67-75

124. Steffe JF (1992) Rheological methods in food processing engineering, 1st edn. Freeman Press, East Lansing

125. Stewart WE, Caracotsios M, Sorensen JP (1992) Parameter estimation from multiresponse data. AICHE J 38(5):641-650

126. Sugisawa $H$ (1966) The thermal degradation of sugars: II. The volatile decomposition products of glucose caramel. J Food Sci 31(3):381-385

127. Terrab A, Gonzalez AG, Diez MJ, Heredia FJ (2003) Characterisation of Moroccan unifloral honeys using multivariate analysis. Eur Food Res Technol 218(1):88-95

128. Togrul H, Arslan N (2004) Mathematical model for prediction of apparent viscosity of molasses. J Food Eng 62(3):281-289

129. Tosun I (2004) Color changes and 5-hydroxymethyl furfural formation in zile pekmezi during storage. Grasas Aceites 55(3):259-263

130. Tzoumaki MV, Biliaderis CG, Vasilakakis M (2009) Impact of edible coatings and packaging on quality of white asparagus (Asparagus officinalis L.) during cold storage. Food Chem 117(1):55-63

131. van Boekel MAJS (1996) Statistical aspects of kinetic modeling for food science problems. J Food Sci 61(3):447-485, 489

132. van Boekel MAJS (2001) Kinetic aspects of the Maillard reaction: a critical review. Nahrung 45(3):150-159

133. Villalobos-Carvajal R, Hernandez-Munoz P, Albors A, Chiralt A (2009) Barrier and optical properties of edible hydroxypropyl methylcellulose coatings containing surfactants applied to fresh cut carrot slices. Food Hydrocolloids 23(2):526-535

134. Vitali AA, Rao MA (1984) Flow properties of low-pulp concentrated orange juice: effect of temperature and concentration. J Food Sci 49(3):882-888

135. Vukov K (1965) Kinetic aspects of sucrose hydrolysis. Int Sugar J $67: 172-175$

136. Williams ML, Landel RF, Ferry JD (1955) The temperature dependence of relaxation mechanisms in amorphous polymers and other glass-forming liquids. J Am Chem Soc 77:3701-3707 